\title{
Importance of charge self-consistency in first-principles description of strongly correlated systems
}

\author{
Swagata Acharya $\mathbb{( D}^{1 凶}$, Dimitar Pashov $\mathbb{D}^{2}$, Alexander N. Rudenko $\mathbb{D}^{1}$, Malte Rösner $\mathbb{D}^{1}$, Mark van Schilfgaarde ${ }^{2,3}$ and \\ Mikhail I. Katsnelson (iD)
}

First-principles approaches have been successful in solving many-body Hamiltonians for real materials to an extent when correlations are weak or moderate. As the electronic correlations become stronger often embedding methods based on firstprinciples approaches are used to better treat the correlations by solving a suitably chosen many-body Hamiltonian with a higher level theory. The success of such embedding theories, often referred to as second-principles, is commonly measured by the quality of self-energy $\Sigma$ which is either a function of energy or momentum or both. However, $\Sigma$ should, in principle, also modify the electronic eigenfunctions and thus change the real space charge distribution. While such practices are not prevalent, some works that use embedding techniques do take into account these effects. In such cases, choice of partitioning, of the parameters defining the correlated Hamiltonian, of double-counting corrections, and the adequacy of low-level Hamiltonian hosting the correlated subspace hinder a systematic and unambiguous understanding of such effects. Further, for a large variety of correlated systems, strong correlations are largely confined to the charge sector. Then an adequate nonlocal low-order theory is important, and the high-order local correlations embedding contributes become redundant. Here we study the impact of charge self-consistency within two example cases, $\mathrm{TiSe}_{2}$ and $\mathrm{CrBr}_{3}$, and show how real space charge re-distribution due to correlation effects taken into account within a first-principles Green's function-based many-body perturbative approach is key in driving qualitative changes to the final electronic structure of these materials.

npj Computational Materials (2021)7:208; https://doi.org/10.1038/s41524-021-00676-5

\section{INTRODUCTION}

Density functional theory ${ }^{1-3}$ has been the workhorse for materialsspecific electronic structure calculations for the last half of the century. Despite enormous success in many respects, it has however some intrinsic limitations. First of all, although the Hohenberg-Kohn theorem ${ }^{1}$ guarantees the existence of some density functional providing an exact ground state energy at a given charge density distribution $\rho$, its exact form is unknown. In practice, this functional is considered as being local or almost local (generalized gradient corrections), which is generally speaking an uncontrollable approximation (for detailed discussions see the review) ${ }^{3}$. Next, and even more importantly, the Kohn-Sham quasiparticles ${ }^{2}$ are, generally speaking, just auxiliary quantities to calculate the total energy and their direct comparison with experimental spectroscopic information is hardly justifiable. Although this is regularly done with partial excellent agreement, there are numerous counterexamples starting from the famous "gap problem" in semiconductors ${ }^{4}$.

An alternative approach is based on the concept of functionals of the Green's function. Luttinger-Ward ${ }^{5}$ and Baym-Kadanoff ${ }^{6}$ theorems respectively prove the existence of such functionals inand out-of-equilibrium. Conceptually, this way is more attractive since the knowledge of an exact single- and two-particle Green's functions guarantees an accurate description of spectroscopic properties of solids ${ }^{7}$. On the other hand, again, an exact form of this functional is practically unknown and we have just its formal definition in terms of infinite sums of skeleton free-leg diagrams $^{5,6}$. If we are interested in a description of subtle phenomena such as, e.g., the Kondo effect ${ }^{8}$ or nonquasiparticle states in half-metallic ferromagnets ${ }^{9}$, the necessary sequence of diagrams seems to be too complicated to be practically taken into account for a complete first-principle realization.

Therefore, alternative embedding approaches were introduced which combine first-principle calculations with model treatments to describe the strong correlations within some low-energy subspace. This way, weakly correlated states at high energies are described within a low-level theory, while the strongly correlated sub-space is treated in higher-level approaches. This is popularly done by mapping the low-energy space to multi-band generalized Hubbard models, which are afterwards often solved, e.g., using dynamical mean-field theory $(\mathrm{DMFT})^{10}$, a program suggested and called 'LDA ${ }^{++\prime}$ in ref. ${ }^{11}$ and which we refer to in the following as 'second principles'. In many cases, this leads to a dramatic improvement of description of strong correlation effects in real materials with itinerant-electron magnets ${ }^{12}$ and heavyfermion compounds ${ }^{13}$ being two major successful examples (for detailed reviews see refs. ${ }^{9,14,15}$ ). Of course, DMFT ${ }^{16}$ is a local approximation that takes only the energy dependence of electronic self energy into account and completely neglects its momentum dependence. However, the latter can be taken into account via various beyond-DMFT diagrammatic approaches ${ }^{17}$, rendering it a technical problem rather than a fundamental one. Also the way how one can map the first-principle electronic structure onto efficient Hamiltonians can be, in principle, improved. The contemporary way is based on the so-called constrained RPA (cRPA) approach ${ }^{18,19}$ but there are no principle obstacles to improve it further if necessary.

A key impediment to second-principles approaches is, however, that multiple energy scales are operative: the high-energy scales

${ }^{1}$ Institute for Molecules and Materials, Radboud University, Nijmegen 6525 AJ, The Netherlands. ${ }^{2}$ Theory and Simulation of Condensed Matter, King's College London, The Strand, London WC2R2LS, UK. ${ }^{3}$ National Renewable Energy Laboratory, Golden, 80401 Colorado, USA. ${ }^{凶}$ email: swagata.acharya@ru.nl 
(a)

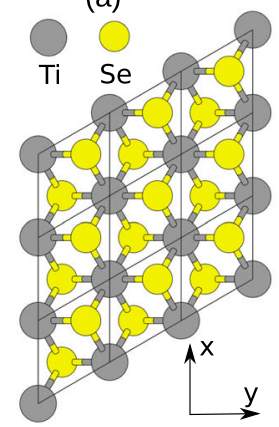

(b)

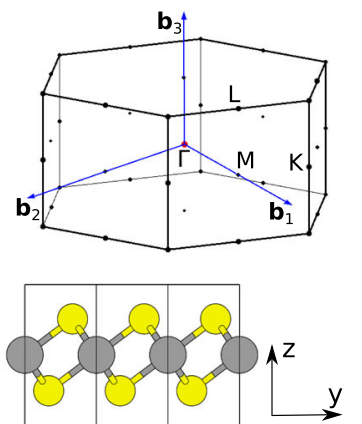

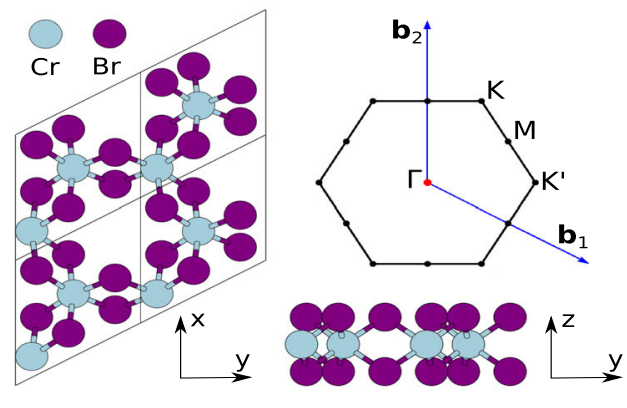

Fig. 1 Ball-and-stick model of the crystal structure, and the Brillouin zone. a Bulk $\mathrm{TiSe}_{2}$ and $\mathbf{b} 1 \mathrm{~L}-\mathrm{CrBr}_{3} . \mathbf{b}_{1}$, $\mathbf{b}_{2}$, and $\mathbf{b}_{3}$ denote reciprocal lattice vectors.

controlling low-energy fluctuations cannot be integrated out without model assumptions. High-energy scales contain information about chemistry and disorder specific to real materials. Yet while first-principles theories contain this information, their application to strongly correlated systems has been limited. In weakly correlated materials, 'first-principles approaches tend to predominate because they rely on a minimum of model assumptions, and are often predictive. This is not the case when correlations are strong because standard methods, usually based on extensions of density functional theory (DFT), lack the sophistication to encapsulate the strong spin and charge fluctuations, or the fidelity to characterize one-particle properties near the Fermi level (which are essential to capture low-energy excitations characteristic of correlated systems); nor are they adequately equipped to generate the (two-particle) suceptibilities. Even in weakly correlated cases, dynamical screening (perhaps the most important many-body effect ${ }^{20}$ ) is not well treated by such standard one-body descriptions ${ }^{21,22}$. The difficulties are even more severe for spin fluctuations, where the characteristic energy scale for excitations can be very small.

Models such as the Hubbard Hamiltonian do indeed contain, within some region of parameter space, key many-body effects such as the metal-insulator transition, pseudogap phases, quantum criticality, and both conventional and unconventional superconductivity. Thus, applications of this model has become the canonical approach to characterizing such phenomena. However, the limits to such an approach become apparent when the high-energy scales that control parameters for the low-energy ones are nontrivial. Furthermore, within any model Hamiltonian, there are only two possibilities for the correlation effects to modify the electronic structure, namely via the energy- and/or the momentum-dependencies of the self-energy $\Sigma$. In first-principle approaches there is, however, an additional possibility in form of the charge self-consistency. That is, if the correlation effects can essentially modify the charge distribution $\rho$, than one needs to recalculate the model Hamiltonian at every iteration which makes the mapping procedure very cumbersome or even practically useless. Several DFT + DMFT works have been published ${ }^{23-32}$ where charge self consistency is performed and shown to make quantitative and/or qualitative changes to the real space charge distribution. However, owing to the lack of internal selfconsistency in choosing the correlated Hamiltonian, the correlations parameters and double counting corrections, systematic and unambiguous understanding of real space charge distribution has been limited. Both DMFT and GW are functionals of the Green's function, and they can be made first-principle. In a recently developed advanced $G_{0} W_{0}+$ DMFT approach ${ }^{33-36}$, authors have surmounted many of the problems that hinder an internal selfconsistency and this is a very promising path for embedding approaches to become truly first principles. Yet many correlated systems, except for a few specific non-perturbative physical situations (typically when spin fluctuations are strong), can be well captured within a many body perturbative framework that is completely free of ambiguities that plague most commonly practiced second-principles approaches, and moreover, the additional high-order correlations are redundant. The physical question of fundamental importance, in such cases, is the following: can a real-space charge redistribution due to correlation effects be qualitatively important leading not just to a moderate renormalization of the model parameters but also to a reconstruction of the electronic structure beyond any purely model consideration? In this work we give a positive answer on this question providing two examples, namely, $\mathrm{TiSe}_{2}$ and $\mathrm{CrBr}_{3}$.

We show in the following how different levels of theory significantly modify the effective one-body potential through changes in the electron density. To this end, we employ three different levels of theory: the local-density approximation (LDA), QSGW theory ${ }^{37-39}$, which, in contrast to conventional GW, modifies the charge density and is determined by a variational principle ${ }^{40}$, and finally an extension of QSGW, where the polarizability needed to construct $W$ is computed including vertex corrections (ladder diagrams) by solving a Bethe-Salpeter equation (BSE) for the two-particle Hamiltonian ${ }^{41}$. We denote the latter QSGW $\widehat{W}$, with the substitution $W \rightarrow \widehat{W}$ signifying that a BSE was solved to compute $W$. In each cycle, the RPA polarizability is made anew, which determines the RPA $W$. In each cycle the four-point polarizability is recomputed from the (newly updated) static part of $W$, to update $\widehat{W}$. These first-principles approaches allow us to carefully analyze the impact of the full charge self consistency taking correlation effects with increasing diagrammatic precision into account.

In terms of diagram classes taken into account QSGW and QS $G \widehat{W}$ represent the forefront of currently available first-principle approaches. As we show, it is essential that the first-principles starting point is of sufficiently high fidelity to capture physics the second-principles scheme cannot reach. First-principles schemes are too cumbersome to handle more than a limited class of diagrams, and it may still be true in general that second-principles schemes may still be needed to capture physics outside the reach of the first-principles scheme. Kondo effect, non-quasi-particle states in weakly doped Mott insulators, Hund's metals, and halfmetallic ferromagnets are archetypal examples. For $\mathrm{TiSe}_{2}$ and $\mathrm{CrBr}_{3}$, however, QSGW/QSG $\widehat{W}$ adequately describes most physical observables, both for its ground- and excited-states ${ }^{42,43}$ obviating the need for higher-order spin-fluctuation diagrams, usually accomplished by second-principles schemes. QSGW and QSGW don't include spin fluctuation diagrams beyond Fock exchange, but these extra diagrams are unimportant for $\mathrm{TiSe}_{2}$, being nonmagnetic and not para-magnetic, and $\mathrm{CrBr}_{3}$, an Ising ferromagnet with a large local moment. The rest of the paper discusses the crucial role of charge self-consistency in such materials, which form an enormous proportion of the condensed matter systems, 
where 'second-principles' approaches and corresponding ambiguities emerging from splitting of the all-electron problem into impurity and bath can be avoided altogether. While selfconsistency may also be also important in some highly correlated materials QSGW cannot address, an analysis of them is beyond the scope of this paper.

\section{RESULTS}

\section{Electronic structure of $\mathrm{TiSe}_{2}$}

$\mathrm{TiSe}_{2}$ is a layered diselenide compound with space group $P \overline{3} m 1$ (Fig. 1). Below 200K, it undergoes a phase transition to a chargedensity wave $(C D W)$, forming a commensurate $2 \times 2 \times 2$ superlattice $(P \overline{3} C 1)$ of the original structure. At the transition there is a softening of the zone boundary phonon accompanied by changes in the transport properties ${ }^{44-49}$.

A number of works have tried to determine the energy dispersion around the Fermi level in both phases with a special focus on the overlap/gap between the Se- $4 p$ valence band at $\Gamma$ and the Ti-3d conduction band at $\mathrm{L}$, sometimes with discordant results. In the high-temperature $P \overline{3} m 1$ phase, reports range from predicting a semimetal (overlap $<120 \mathrm{meV}$ ) between these bands, to an insulator with $<60$ meV gap, depending on the study ${ }^{50-57}$. In the CDW $P \overline{3} C 1$ phase there is a greater consensus, namely that the gap is small and positive. In brief, upon cooling the system, the CDW transition induces a distortion that either (slightly) increases the existing gap or leads to a gap opening between these bands with the gap for the CDW phase being 100-150 $\mathrm{meV}^{50,53-55,57}$.

At high temperature, the positive or negative (i.e., overlap) indirect gap between Se-4p and Ti-3d bands is larger than the negative gap obtained with standard DFT calculations. In fact, DFT is not helpful because it predicts a negative gap in both the undistorted and CDW phases. Bianco et al. ${ }^{58}$ finds with LDA $+U(U=3.9 \mathrm{eV})$ a gap of $14 \mathrm{meV}$ in the $P \overline{3} \mathrm{~m} 1$ phase and $200 \mathrm{meV}$ in the CDW phase. LDA $+U$ is a kind of 'second-principles' method because the answer depends $U$, which is not known. To check whether the negative gap is merely an artifact of the model, Cazzaniga et al. ${ }^{59}$ considered a $G_{0} W_{0}$ calculation based on the LDA, and found a gap of $\sim 200 \mathrm{meV}$ in the high-temperature $P \overline{3} m 1$ structure.

We show here that while $G W$ does indeed modify the quasiparticle spectrum, the true situation is more complex. This is because not only the eigenvalues but the density is significantly renormalized relative to the LDA. This induces a corresponding change in the effective potential through the inverse of the susceptibility, $X^{-1}\left(x_{1}, x_{2}\right)=\delta V\left(x_{1}\right) / \delta n\left(x_{2}\right)$. What appears to be special about $\mathrm{TiSe}_{2}$ is that $X^{-1}(1,2)$ is large, and the correction to the LDA density modifies the effective potential $V$ in such a way as to reduce the splitting between occupied and unoccupied levels. This is very unusual: it has long been established that in the vast majority of cases, GW based on the LDA continues to underestimate the gap in semiconductors, albeit less so than the LDA ${ }^{60}$

These effects can only be found through self-consistency. QSGW is ideally suited for this case, as its excitation spectra are generally superior to fully self-consistent $G W^{61-65}$. We find that the $P \overline{3} m 1$ is indeed semimetallic, as is the case with DFT, but for different reasons. We first revisit the $G W$ calculation of the undistorted $P \overline{3} c 1$ structure, but with some modifications:

- we did not include a $Z$ factor. There are various justifications for this, most notably as an approximate way to incorporate self-consistency in $G$ with fixed $W$; see Appendix in ref. ${ }^{60}$. Omission of $Z$ tends to widen bandgaps.

- the full matrix $G^{\mathrm{LDA}} W^{\mathrm{LDA}}$ is used, in the QSGW sense ${ }^{38}$ :

$$
\left.\Sigma_{i j}^{0}=\frac{1}{2} \sum_{i j}\left|\psi_{i}\right\rangle\left\{\operatorname{Re}\left[\Sigma\left(\varepsilon_{i}\right)\right]_{i j}+\operatorname{Re}\left[\Sigma \varepsilon_{j}\right)\right]_{i j}\right\}\left\langle\psi_{j}\right|
$$

Panel (a) of Fig. 2 shows LDA and $G^{\mathrm{LDA}} W^{\mathrm{LDA}}$ bands similar to the $G^{\mathrm{LDA}} W^{\mathrm{LDA}}$ calculation of ref. ${ }^{59}$. Focusing on the LDA bands, the highest occupied state at $\Gamma$ turns red very close to $\Gamma$, indicating the penetration of the Ti-derived conduction band into the valence band (indicating a 'negative' direct gap). This is an artifact of the LDA's well known tendency to underestimate splittings between occupied and unoccupied states, and $G^{\mathrm{LDA}} W^{\mathrm{LDA}}$ increases this separation (blue dashed lines) as it typically does. The (indirect) $G^{\mathrm{LDA}} W^{\mathrm{LDA}}$ gap of $300 \mathrm{meV}$ is slightly larger than ref. ${ }^{59}$, in line with the unit $Z$ factor used in the present calculation.

Figure $2 \mathrm{~b}$ shows that self-consistency is crucially important in $\mathrm{TiSe}_{2}$. The off-diagonal elements of $\Sigma_{i j}^{0}$ modify the density $n(\mathbf{r})$ and thus $V(\mathbf{r})$. A simple way to estimate $\Delta V$ is to make an ansatz that the LDA adequately yields $X^{-1}=\delta V / \delta n$. For a modified $\bar{n}$ the potential becomes $V(\bar{n})=\Sigma^{0}-V_{\mathrm{xc}}\left(n^{\mathrm{LDA}}\right)+V_{\mathrm{xc}}(\bar{n}) . \bar{n}$ can be determined self-consistently in the usual manner by adding a fixed external potential $\Sigma^{0}-V_{x c}\left(n^{\mathrm{LDA}}\right)$ to the LDA Hamiltonian and allowing it to go self-consistent. Remarkably, the gap becomes negative again, as shown by the blue dashed lines in Fig. $2 b$, but the dispersion is very different from the LDA. In particular the inverted gap character at $\Gamma$ disappears, which is topologically essential for a gap to form at $\Gamma$. The quality of the ansatz can be checked by carrying out a complete QSGW calculation. This is shown as solid lines in Fig. 2b, and it demonstrates the ansatz is reasonable. As we will show elsewhere, the experimentally observed low-temperature gap forms as a consequence of the charge density-wave instability.

\section{Electronic structure of $\mathrm{CrBr}_{3}$}

Monolayer (1L) of $\mathrm{CrBr}_{3}$ is a two-dimensional ferromagnetic (FM) insulator where the magnetic moments of monolayer $\mathrm{CrBr}_{3}$ align normal to the plane (see Fig. 1 for the crystal structure). The spontaneous magnetization persists in monolayer $\mathrm{CrBr}_{3}$ with a
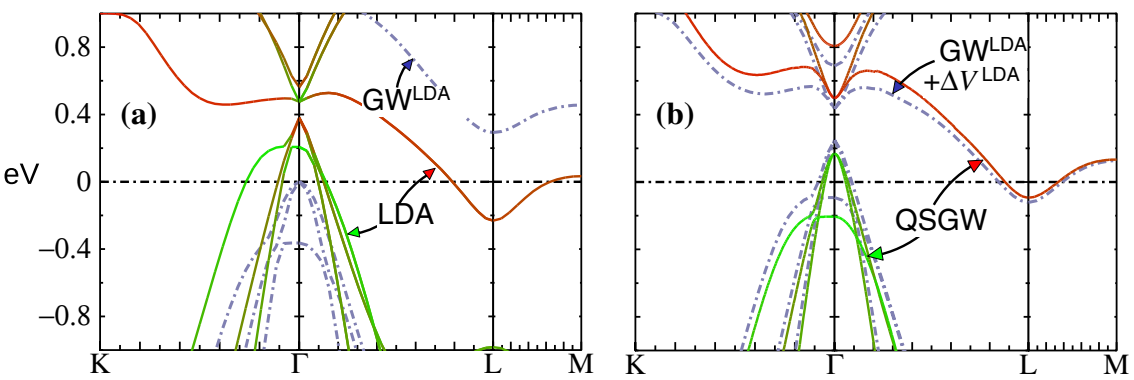

Fig. 2 Energy bands of the undistorted $\bar{P} \overline{\mathbf{3}} \boldsymbol{C} 1$ structure. a Solid lines are LDA results, with red and green depicting a projection onto Ti and Se orbital character, respectively. Blue dashed line shows shifts calculated in the GW approximation based on the LDA, as described in the text. b Blue dashed line shows results from GW based on the LDA (same $\Sigma$ as in panel (a)), with an extra potential $\Delta V^{\mathrm{LDA}}$ deriving from a $\rho$ shift computed from the rotation of the LDA eigenvectors. Solid lines are QSGW results, with the same color scheme as in panel (a). 
Curie temperature of $34 \mathrm{~K}^{66}$. Within a purely atomic picture, fully determined by the crystal field environment and the Hund's multiplet structure ${ }^{67}$, the low-energy properties of the materials and the magnetism should be entirely governed by $\mathrm{Cr}-d$ electrons. However, the ligands, their masses, and the number of core states they have, play an integral role in determining the low-energy properties of $\mathrm{CrBr}_{3}$. In a separate work we discuss the role of the ligands like $\mathrm{Cl}, \mathrm{Br}$ and $\mathrm{I}$ in determining the crucial low-energy properties of the entire class of $1 \mathrm{~L}$ Chromium trihalides ${ }^{42}$. The $\mathrm{Br}-p$ states strongly hybridize with $\mathrm{Cr}-d$ states in $\mathrm{CrBr}_{3}$. In the present work we show how charge self-consistency at different levels of the theory controls the nature of the eigenfunctions and the $\mathrm{Br}$ component in the valence band manifold of $1 \mathrm{~L} \mathrm{CrBr}_{3}$.

We simulate the free standing $1 \mathrm{~L}$ of ferromagnetic $\mathrm{CrBr}_{3}$ within LDA, QSGW and QSGW. We also perform a rigorous check for vacuum correction to all relevant observables by increasing the vacuum size from 20 to $80 \AA^{42}$. We check for convergence and scaling of band gap and the dielectric constant $\epsilon_{\infty}$ with vacuum size as discussed in a separate work ${ }^{42}$. We observe that FM-1L $\mathrm{CrBr}_{3}$ is an insulator with $1.3 \mathrm{eV}$ of electronic band gap in LDA, which is significantly lower than in QSGW yielding a gap of $5.7 \mathrm{eV}$. The large enhancement in QSGW band gaps relative to the LDA is standard in polar compounds ${ }^{37}$. Nevertheless, within the random phase approximation (RPA), it has long been known that $W$ is universally too large ${ }^{68,69}$, which is reflected in an underestimate of the static dielectric constant $\epsilon_{\infty}$. Empirically, $\epsilon_{\infty}$ seems to be underestimated in QSGW by a nearly universal factor of $0.8^{70}$, for a wide range of insulators ${ }^{71,72}$ resulting in slightly overestimated ${ }^{37}$ band gaps. This can be corrected by extending the RPA screening to introduce an electron-hole attraction in virtual excitations. These extra (ladder) diagrams are solved by a BSE, and they significantly improve the optics and largely eliminating the discrepancy in $\epsilon_{\infty}{ }^{73}$. When ladder diagrams are also added to improve $W$ in the $G W$ cycle $(W \rightarrow \widehat{W})$, it significantly improves the one-particle gap as well ${ }^{41,74}$. In detail, our QSGW implementation is self-consistent in the sense that the updated $\widehat{W}$ also updates $\Sigma$ and hence the cycle continues until $\widehat{W}, \Sigma$ and $G$ converge iteratively. This scenario is played out in $\mathrm{CrBr}_{3}$ : the QSGW bandgap is slightly larger than QSGW bandgap, as seen in Table 1. Also we converge the observables like band-gap and $\epsilon_{\infty}$ by increasing the size of the two-particle Hamiltonian within our self-consistent BSE implementation. We find that for $\mathrm{CrBr}_{3}$ to converge both observables we find it necessary to include 24 valence bands and 14 conduction bands in two-particle Hamiltonian that we solve within BSE. Twenty-four valence bands are effectively all bands that emerge from hybridization between occupied $\mathrm{Cr}-\mathrm{d}-\mathrm{t}_{2 g \uparrow}$

Table 1. Fraction of spectral weight that the Halogen ( $\mathrm{Br}$ ) contributes to the total within an energy window of 0 (Fermi energy) to $0.6 \mathrm{eV}$ below the Fermi energy (bound states).

\begin{tabular}{lllllll}
\hline variants & LDA & QSGW & QSGW & $\Sigma^{\mathrm{QSGW}}[\rho(\mathrm{LDA})]$ & $\Sigma^{\mathrm{QSGW}}[\rho(\mathrm{QSG} \widehat{W})]$ & $\Sigma^{\mathrm{QSGW}}[\rho(\mathrm{QSGW})]$ \\
\hline$\%$ of $\mathrm{Br}$ & 31 & 69 & 37 & 23 & 58 & 47 \\
$\mathrm{Cr}-\boldsymbol{d}$ & 4.44 & 4.3 & 4.35 & 4.36 & 4.32 & 4.31 \\
gap $(\mathrm{eV})$ & 1.3 & 5.7 & 4.65 & 6.0 & 5.7 & 4.69 \\
\hline
\end{tabular}

The $\mathrm{Cr}-d$ occupancies, and the electronic band gaps with different choices of self consistent $\rho$ and $\Sigma$ are shown.

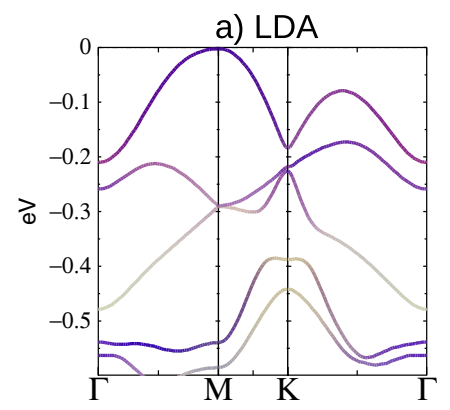

d) $\sum{ }^{Q S G W} \rho^{L D A}$

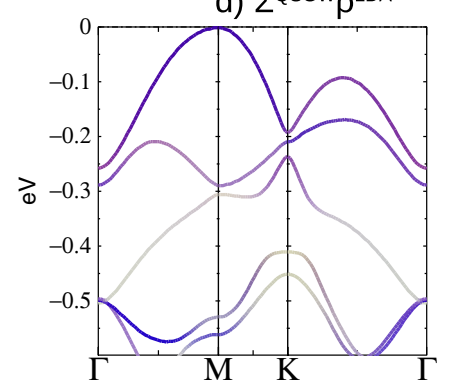

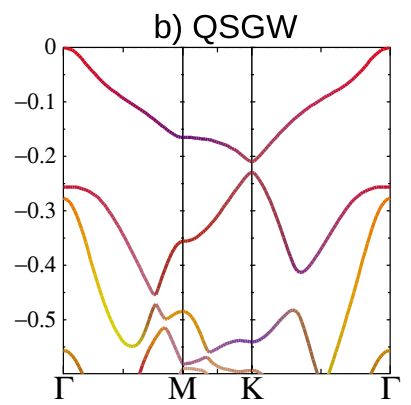

e) $\Sigma^{Q S G W} \rho^{Q S G W}$

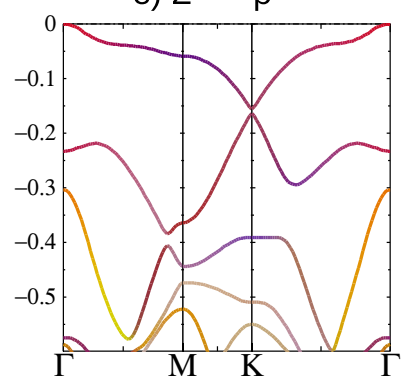

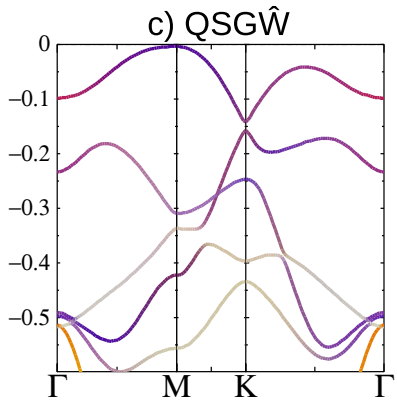

f) $\Sigma^{Q S G W} \rho^{Q S G W}$

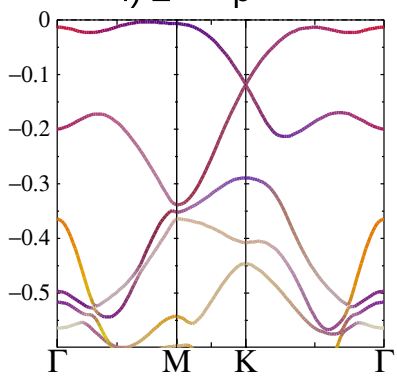

Fig. 3 Electronic band structure of $\mathrm{CrBr}_{3}$. a LDA, b QSGW, and c QSGW. $\widehat{W}$, we mean the polarizability that enters into $W$ is calculated beyond the time-dependent Hartree approximation (RPA) but also includes ladder diagrams computed from a four-point Bethe-Salpeter equation (BSE). This significantly improves $W$ as seen by comparing the macroscopic dielectric function to experiment. Colors correspond to $\mathrm{Br}-p_{x}+p_{y}$ (red), $\mathrm{Br}-p_{z}$ (green), $\mathrm{Cr}-d$ (blue). A fourth color (white) selects the $\mathrm{Cr}-\mathrm{e}_{g}$ manifold, and washes out the color to the extent it is present. Topmost valence bands of LDA and BSE have similar shape, apart from a strong narrowing the bandwidth. The shape changes in QSGW. with the VBM shifting to $\Gamma$. (d) $\Sigma^{\mathrm{QSGW}}[\rho(\mathrm{LDA})]$, (e) $\Sigma^{\mathrm{QSGW}}[\rho(\mathrm{QSGW})]$, and (f) $\Sigma^{\mathrm{QSGW}}[\rho(\mathrm{QSGW})]$. 

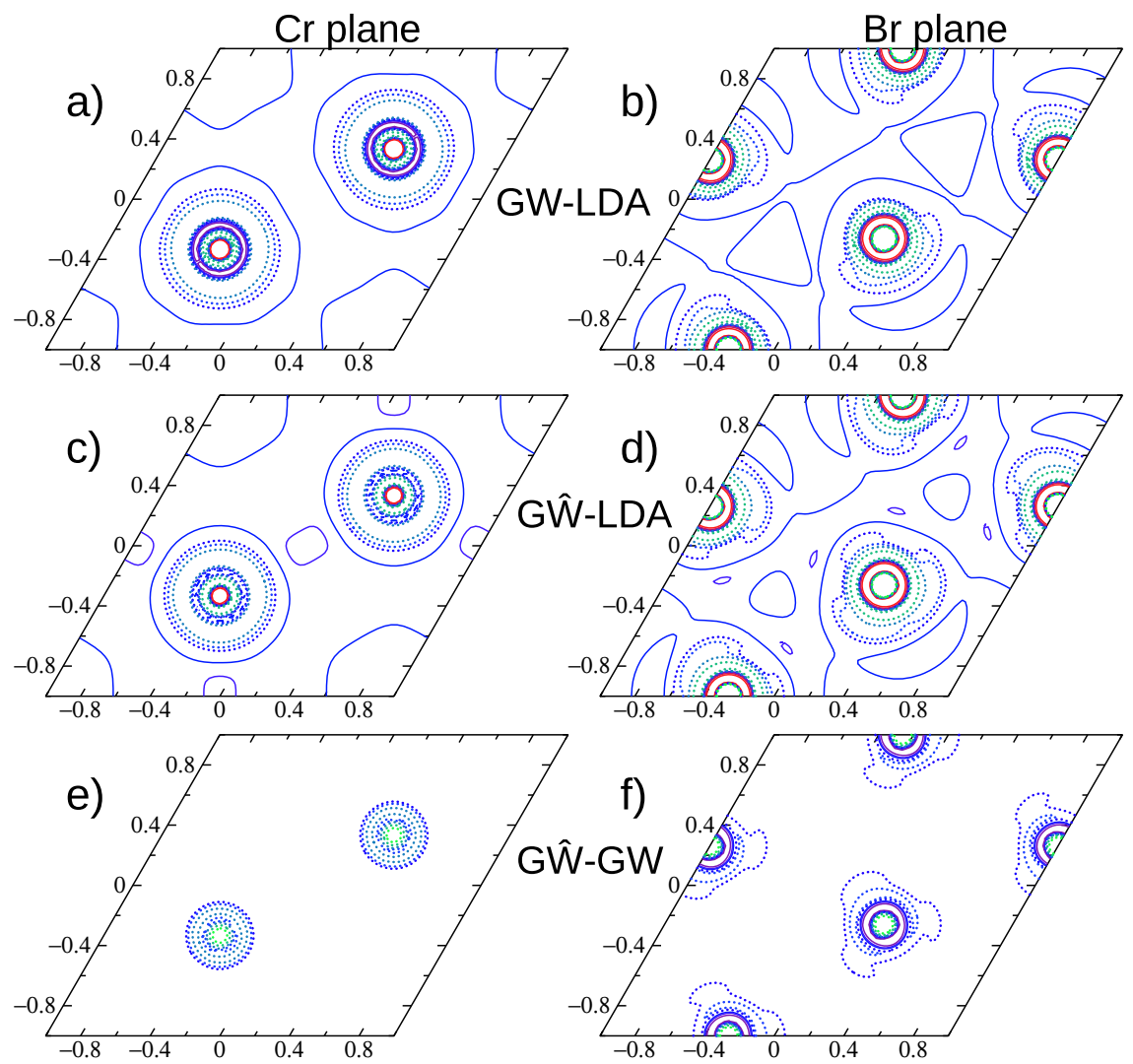

Fig. 4 Real-space charge densities $(\rho)$ of $\mathrm{CrBr}_{3}$ of the highest valence band state with the abscissa and ordinate $x$ and $y$. All the left panels $(\mathbf{a}, \mathbf{c}, \mathbf{e})$ pass through a $\mathrm{Cr}$ plane and right panels $(\mathbf{b}, \mathbf{d}, \mathbf{f})$ pass through a Br plane. Panels $(\mathbf{a}, \mathbf{b})$ display constant-amplitude contours for $\mathrm{QSGW} \rho$ after subtracting out the LDA $\rho$. Contours are taken in half-decade increments in $\rho$, with a factor of 300 between highest contour (red) and lowest (blue). Panels (c, d) show the change in $\rho$ passing from LDA to QSGW eigenfunctions; panels (e, $\mathbf{f}$ ) show the corresponding change passing from QSGW to QSGW. In the bottom four panels $(\mathbf{c}-\mathbf{f})$, blue $\rightarrow$ red has a similar meaning as in the top panels (increasing positive $\delta \rho$ ), while contours of negative $\delta \rho$ are depicted by increasing strength in the change blue $\rightarrow$ green.

orbitals ( 3 from each $\mathrm{Cr}$, totaling 6 for $2 \mathrm{Cr}$ atoms in the unit cell) and $\mathrm{Br}-\mathrm{p}$ orbitals (total 18 states from $6 \mathrm{Br}$ atoms in the unit cell). Fourteen conduction states comprise all the unoccupied $\mathrm{Cr}-\mathrm{d} \mathrm{t}_{2 g \downarrow}$ and $\mathrm{e}_{g \downarrow}$ states. Larger sizes of the two-particle Hamiltonian do not lead to any significant changes in the optical properties. The convergence with respect to the number of states entering into the two-particle is significantly slower than in simple $s p$ semiconductors, which reflects the Frenkel-like character of the electron-hole attraction. We also converge the observables in terms of (artificial) vacuum size.

Next we examine independent variations of the Hartree potential, via the density $\rho$, and the self-energy $\Sigma^{0}$. In the $G W$ case, $\Sigma^{0}$ denotes the quasiparticlized version of the dynamical self-energy $\Sigma(\omega)$; for the LDA it denotes the LDA exchangecorrelation potential. Unless stated otherwise, results are presented with $\rho$ and $\Sigma^{0}$ internally self-consistent. Considering this case at first, there is a remarkable difference between the LDA and QSGW electronic band structures. Within LDA (see Fig. 3a), the valence band maximum falls at the $M$ point, while within QSGW it shifts to the $\Gamma$ point (see Fig. 3b). The eigenfunctions are also quite different: the $\mathrm{Br}$ contribution to the low-energy valence band manifold is significantly larger within QSGW. At a still higher level of theory replacing $W \rightarrow \widehat{W}$, a portion of the strong perturbation of the LDA band structure is partially undone (Fig. 3c); shifts $\mathrm{Br}$ contribution to the valence eigenfunctions in the direction of the LDA (see Table 1). This is readily understood as a softening of $W$ by the ladder diagrams, as noted above. With the QSGW, the bandgap in $\mathrm{CrBr}_{3}$ is reduced slightly to $4.65 \mathrm{eV}$. The top most valence band in QSGW has a shape similar to LDA but the band gap is approximately three times as large and the valence bandwidth gets renormalized by a factor of $\sim 2$. The observation that QSGW more closely resembles the LDA than QSGW is remarkable and calls for further analysis.

To this end, we consider independent variations of $\rho$ and $\Sigma^{0}$ in the following senses:

- $\rho$ from LDA and $\Sigma^{0}$ from QSGW, which we denote as $\Sigma^{\mathrm{QS} G W}[\rho$ (LDA)]. This scheme produces a valence band structure similar to LDA (Fig. 3d), but with $6.0 \mathrm{eV}$ electronic gap, close to the QSGW gap of $5.7 \mathrm{eV}$. This clearly establishes the important role of the density in determining the effective one-body hamiltonian. As in the case of simple $s p$ tetrahedral semiconductors where the LDA density is already rather good, the gap change is mainly controlled by the nonlocality in the self-energy which the LDA misses ${ }^{75,76}$.

- $\rho$ from QSGW and $\Sigma$ from QSGW, which we denote as $\Sigma^{\mathrm{QSGW}}[\rho(\mathrm{QSGW})]$ (Fig. 3e). Now the valence band structure is much closer to the QSGW band structure, although the top most valence band is significantly narrowed. Also, the gap is similar to QSGW gap $(5.7 \mathrm{eV})$. This tells us that the Hartree and many-body contributions cannot be decoupled. The addition of electron-hole ladder diagrams should considerably improve on the RPA's known inadequacy in describing short-ranged correlations ${ }^{77}$, and here we see that it affects both Hartree and exchange-correlation parts. 
- $\rho$ from QSGW and $\Sigma$ from QSGW, which we denote as $\Sigma^{\mathrm{QS} G W}[\rho(\mathrm{QSGW})]$ (bottom right panel of Fig. 3f). This shows in a different way how the Hartree and correlation contributions to the potential are interwined.

To further probe the role of the charge density, we plot $\rho$ in the planes passing through the $\mathrm{Cr}$ and $\mathrm{Br}$ atoms at different levels of theory (Fig. 4). The density is plotted in real space, and the abscissa and ordinate are defined by the inverse transpose of the $2 \times 2$ matrix composed of $\mathbf{b}_{1}$ and $\mathbf{b}_{\mathbf{2}}$ (Fig. 1) with $x$ and $y$ defined by aligning $\mathbf{b}_{\mathbf{2}}$ parallel to $y$. In this notation, the $M$ point is on the $\mathbf{b}_{\mathbf{2}}$ line, or the $y$ axis. On the formation of the $2 \mathrm{D}$ crystal charge is augmented on the $\mathrm{Cr}-\mathrm{Cr}$ and $\mathrm{Br}-\mathrm{Br}$ bonds, taking it away from the atoms. QSGW accentuates this tendency (Fig. 4a, b), as does QS $G \widehat{W}$, but to a relatively lesser extent (Fig. $4 c$, d). However, although the structure of the top most valence band seems similar within LDA and QSGW and different within QSGW and QSGW, we show in Fig. $4 \mathrm{e}, \mathrm{f}$ that the real-space $\rho(Q S G \widehat{W})$ is much closer to $\rho(Q S G W)$ compared to LDA. In short, QSGW weakly modifies and slightly localizes charges in comparison to QSGW. In a separate work $^{42}$ we have shown that there is a generic tendency across this entire class of systems $\mathrm{CrX}_{3}$ to realize a very different Cr- $d$-X-p hybridization within QSGW compared to QSGW. We added a small $U$ of $1 \mathrm{eV}$ to the $\mathrm{Cr}-d$ in QSGW and allowed further charge and $\Sigma$ self-consistency and, curiously enough, it reproduces the QSGW electronic band structure. Applying $U$ shifts the $\mathrm{Cr}$ majority spin down and the minority spin up and leads to an increase is hybridization with the Br-p. The key takeaway is that the QSGW solution is same as the self consistent solution from QS $G \widehat{W}+U$. This is expected as the ladder in the QSGW screens the W compared to QSGW. However, the addition of $U$ to our selfconsistent QSGW ability not only changes the one-particle eigenvalues, also changes its eigenfunctions.

\section{DISCUSSION}

In conclusion, using a self-consistent first principles Green's function approach we show how correlations induce large changes in both the one-body (Hartree) and many-body contribution to the potentials, and that the two are inherently intertwined. To demonstrate the effect we considered two currently popular materials systems: a three-dimensional chargedensity-wave candidate $\mathrm{TiSe}_{2}$ and a two-dimensional ferromagnet $\mathrm{CrBr}_{3}$. Such changes to the electronic wavefunction go way beyond any weak renormalization of the parameters that determine the electronic structure within a second-principles approach and thus calls for the development of better firstprinciples approaches that solve many-body Hamiltonians for real materials with better approximations.

\section{METHODS}

\section{One-particle basis functions}

The one-body part of the calculations are performed in an augmentedwave basis whose envelope functions consist of smooth Hankel functions (convolutions of generalized Gaussian functions and Hankel functions). This basis is a generalization of the classic LMTO method of Andersen ${ }^{78}$, with more flexibility, better accuracy, and adapted to a full potential framework. It also has a unique augmentation scheme that converges much more rapidly with augmentation l-cutoff than conventional augmented-wave methods. Details are presented in ref. ${ }^{39}$. For all calculations we used a uniform I cutoff of 4. For $\mathrm{CrBr}_{3}$ we used a basis of spdfspd augmented envelope functions on each $\mathrm{Cr}, \mathrm{Br}$, Ti and Se site. Smooth Hankel energies were set to -0.3 and $-1.1 \mathrm{eV}$, and $\mathrm{l}$-dependent smoothing radii determined by fitting envelope functions to numerical free atom wave functions. For $\mathrm{Cr}$ and $\mathrm{Ti}$ we also included $3 p$ extended and $4 d$ conventional local orbitals. Convergence studies wrt basis size are presented in ref. ${ }^{60}$ for several materials.

\section{QSGW calculations}

The Quasiparticle Self-Consistent GW approximation was introduced in ref. 79 and our all-electron, augmented-wave implementation of it described in detail in ref. ${ }^{38}$. For the plane wave part, we used a coulomb cutoff of 4.4 Ry; for the augmentation part, we used an I-cutoff of $I=8$. The frequency mesh had a spacing of 0.02 Ry for small frequency $\omega$, the spacing increasing linearly with $\omega$.

The one-body calculations for the kinetic energy, band structure, and charge density were performed in a $16 \times 16 \times 16\left(\mathrm{TiSe}_{2}\right)$ and $16 \times 16 \times 1$ $\left(\mathrm{CrBr}_{3}\right) k$-mesh while the (relatively smooth) dynamical self-energy $\Sigma(k)$ was constructed using a $8 \times 8 \times 8\left(\mathrm{TiSe}_{2}\right)$ and $6 \times 6 \times 1\left(\mathrm{CrBr}_{3}\right) k$-mesh and $\Sigma^{\circ}(\mathrm{k})$ extracted from it. For each iteration in the QSGW self-consistency cycle, the charge density was made self-consistent. The QSGW cycle was iterated until the RMS change in $\Sigma^{0}$ reached $10^{-5}$ Ry. Thus the calculation was selfconsistent in both $\Sigma^{0}(k)$ and the density. Numerous checks were made to verify that the self-consistent $\Sigma^{0}(k)$ was independent of starting point, for both QSGW and QSGW calculations; e.g., using LDA or Hartree-Fock selfenergy as the initial self energy for QSGW and using LDA or QSGW as the initial self-energy for QSGW.

\section{Self-consistent ladder-BSE, QSGW, calculations}

For the present work, the electron-hole two-particle correlations are incorporated within a self-consistent ladder-BSE implementation ${ }^{41,73}$ with Tamm-Dancoff approximation ${ }^{80,81}$. The effective interaction $W$ is calculated with ladder-BSE corrections and the self energy, using a static vertex in the BSE. $G$ and $W$ are updated iteratively until all of them converge and this is what we call QSGW. Ladders increase the screening of $W$, reducing the gap besides softening the LDA $\rightarrow$ QSGW corrections noted for the valence bands.

For $\mathrm{CrBr}_{3}$, we checked the convergence in the $\mathrm{QSG} \widehat{W}$ band gap by increasing the size of the two-particle Hamiltonian. We increase the number of valence and conduction states that are included in the twoparticle Hamiltonian. We observe that for all materials the QSGW band gap stops changing once 24 valence and 14 conduction states are included in the two-particle Hamiltonian.

\section{DATA AVAILABILITY}

All input/output data can be made available on reasonable request. All the input file structures and the command lines to launch calculations are rigorously explained in the tutorials available on the Questaal webpage https://www.questaal.org/get/.

\section{CODE AVAILABILITY}

The source codes for LDA, QSGW, and QSGW are available from https://www. questaal.org/get/under the terms of the AGPLv3 license.

Received: 24 June 2021; Accepted: 19 November 2021; Published online: 20 December 2021

\section{REFERENCES}

1. Hohenberg, P. \& Kohn, W. Inhomogeneous electron gas. Phys. Rev. 136, B864-B870 (1964).

2. Kohn, W. \& Sham, L. J. Self-consistent equations including exchange and correlation effects. Phys. Rev. 140, A1133-A1138 (1965).

3. Jones, R. O. \& Gunnarsson, O. The density functional formalism, its applications and prospects. Rev. Mod. Phys. 61, 689 (1989).

4. Aryasetiawan, F. \& Gunnarsson, O. The GW method. Rep. Prog. Phys. 61, 237-312 (1998).

5. Luttinger, J. M. \& Ward, J. C. Ground-state energy of a many-fermion system. ii. Phys. Rev. 118, 1417-1427 (1960).

6. Baym, G. \& Kadanoff, L. P. Conservation laws and correlation functions. Phys. Rev. 124, 287-299 (1961).

7. Nozières, P. Theory of interacting Fermi systems. (Benjamin, New York, 1964).

8. Hewson, A. C. The Kondo Problem to Heavy Fermions (Cambridge Univ. Press, 1993).

9. Katsnelson, M. I., Irkhin, V. Y., Chioncel, L. \& Lichtenstein, A. I. Half-metallic ferromagnets: from band structure to many-body effects. Rev. Mod. Phys. 80, 315 (2008).

10. Anisimov, V. I., Poteryaev, A. I., Korotin, M. A., Anokhin, A. O. \& Kotliar, G. Firstprinciples calculations of the electronic structure and spectra of strongly 
correlated systems: dynamical mean-field theory. J. Phys: Condens. Matter 9 7359-7367 (1997).

11. Lichtenstein, A. I. \& Katsnelson, M. I. Ab initio calculations of quasiparticle band structure in correlated systems: Lda ++ approach. Phys. Rev. B 57, 6884-6895 (1998).

12. Lichtenstein, A. I., Katsnelson, M. I. \& Kotliar, G. Finite-temperature magnetism of transition metals: an ab initio dynamical mean-field theory. Phys. Rev. Lett. 87, 067205 (2001).

13. Choi, H. C., Min, B. I., Shim, J. H., Haule, K. \& Kotliar, G. Temperature-dependent Fermi surface evolution in heavy fermion Celrln 5 . Phys. Rev. Lett. 108, 016402 (2012).

14. Held, K. Electronic structure calculations using dynamical mean field theory. Adv. Phys. 56, 829-926 (2007).

15. Kotliar, G., Savrasov, S. Y., Haule, K., Oudovenko, V. S. \& Parcollet, O. Electronic structure calculations with dynamical mean-field theory. Rev. Mod. Phys. 78, 865 (2006).

16. Georges, A., Kotliar, G., Krauth, W. \& Rozenberg, M. J. Dynamical mean-field theory of strongly correlated fermion systems and the limit of infinite dimensions. Rev. Mod. Phys. 68, 13 (1996).

17. Rohringer, G. et al. Diagrammatic routes to nonlocal correlations beyond dynamical mean field theory. Rev. Mod. Phys. 90, 025003 (2018).

18. Aryasetiawan, F. F., Imada, M., Georges, A., Kotliar, G., Biermann, S. \& Lichtenstein, A. I. Frequency-dependent local interactions and low-energy effective models from electronic structure calculations. Phys. Rev. B 70, 195104 (2004).

19. Honerkamp, C., Shinaoka, H., Assaad, F. F. \& Werner, P. Limitations of constrained random phase approximation downfolding. Phys. Rev. B 98, 235151 (2018).

20. Martin, R. M. Electronic Structure (Cambridge University Press, 2004).

21. Neaton, J. B., Hybertsen, M. S. \& Louie, S. G. Renormalization of molecular electronic levels at metal-molecule interfaces. Phys. Rev. Lett. 97, 216405 (2006).

22. Bruneval, F., Vast, N., Reining, L., Izquierdo, M., Sirotti, F. \& Barrett, N. Exchange and correlation effects in electronic excitations of $\mathrm{Cu}_{2} \mathrm{O}$. Phys. Rev. Lett. 97 267601 (2006).

23. Aichhorn, M., Pourovskii, L. \& Georges, A. Importance of electronic correlations for structural and magnetic properties of the iron pnictide superconductor LaFeAsO. Phys. Rev. B 84, 054529 (2011).

24. Bhandary, S., Assmann, E., Aichhorn, M. \& Held, K. Charge self-consistency in density functional theory combined with dynamical mean field theory: $k$-space reoccupation and orbital order. Phys. Rev. B 94, 155131 (2016).

25. Schüler, M. Charge self-consistent many-body corrections using optimized projected localized orbitals. J. Phys: Condens. Matter 30, 475901 (2018).

26. Hampel, A., Beck, S. \& Ederer, C. Effect of charge self-consistency in DFT + DMFT calculations for complex transition metal oxides. Phys. Rev. Res. 2, 033088 (2020).

27. Bhandary, S., Assmann, E., Aichhorn, M. \& Held, K. Charge self-consistency in density functional theory combined with dynamical mean field theory: $k$-space reoccupation and orbital order. Phys. Rev. B 94, 155131 (2016).

28. Sakuma, R., Werner, P. \& Aryasetiawan, F. Electronic structure of $\mathrm{SrVO}_{3}$ within $\mathrm{GW}$ +DMFT. Phys. Rev. B 88, 235110 (2013).

29. Savrasov, S. Y. \& Kotliar, G. Spectral density functionals for electronic structure calculations. Phys. Rev. B 69, 245101 (2004).

30. Pourovskii, L. V., Amadon, B., Biermann, S. \& Georges, A. Self-consistency over the charge density in dynamical mean-field theory: a linear muffin-tin implementation and some physical implications. Phys. Rev. B 76, 235101 (2007).

31. Di Marco, I. et al. Correlation effects in the total energy, the bulk modulus, and the lattice constant of a transition metal: combined local-density approximation and dynamical mean-field theory applied to $\mathrm{Ni}$ and Mn. Phys. Rev. B 79, 115111 (2009).

32. Grånäs, O. et al. Charge self-consistent dynamical mean-field theory based on the full-potential linear muffin-tin orbital method: methodology and applications. Comput. Mater. Sci. 55, 295-302 (2012).

33. Boehnke, L., Nilsson, F., Aryasetiawan, F. \& Werner, P. When strong correlations become weak: consistent merging of GW and DMFT. Phys. Rev. B 94, 201106 (2016).

34. Nilsson, F., Boehnke, L., Werner, P. \& Aryasetiawan, F. Multitier self-consistent GW + EDMFT. Phys. Rev. Mater. 1, 043803 (2017)

35. Petocchi, F., Nilsson, F., Aryasetiawan, F. \& Werner, P. Screening from $e_{g}$ states and antiferromagnetic correlations in $d^{(1,2,3)}$ perovskites: a GW + EDMFT investigation. Phys. Rev. Res. 2, 013191 (2020).

36. Petocchi, F., Christiansson, V., Nilsson, F., Aryasetiawan, F. \& Werner, P. Normal state of $\mathrm{Nd}_{1-x} \mathrm{Sr}_{x} \mathrm{NiO}_{2}$ from self-consistent GW + EDMFT. Phys. Rev. X 10, 041047 (2020).

37. van Schilfgaarde, M., Kotani, T. \& Faleev, S. Quasiparticle self-consistent GW theory. Phys. Rev. Lett. 96, 226402 (2006).

38. Kotani, T., van Schilfgaarde, M. \& Faleev, S. V. Quasiparticle self-consistent GW method: a basis for the independent-particle approximation. Phys. Rev. B 76, 165106 (2007).
39. Pashov, D. Questaal: a package of electronic structure methods based on the linear muffin-tin orbital technique. Comp. Phys. Comm. 249, 107065 (2020).

40. Ismail-Beigi, S. Justifying quasiparticle self-consistent schemes via gradient optimization in Baym-Kadanoff theory. J. Phys.: Condens. Matter 29, 385501 (2017).

41. Cunningham, B. Gruening, M., Pashov, D. \& van Schilfgaarde, M. QSGW: quasiparticle self consistent GW with ladder diagrams in W. Preprint at https://arxiv. org/abs/2106.05759 (2021).

42. Acharya, S. Electronic structure of chromium trihalides beyond density functional theory. Phys. Rev. B 104, 155109 (2021).

43. Acharya, S. et al. Excitons in bulk and layered chromium tri-halides: from Frenke to the Wannier-Mott limit. Preprint at https://arxiv.org/abs/2110.08174 (2021).

44. Salvo, F. J. D., Moncton, D. E. \& Waszczak, J. V. Electronic properties and superlattice formation in the semimetal TiSe ${ }_{2}$. Phys. Rev. B 14, 4321 (1976).

45. Holt, M., Zschack, P., Hong, H. \& Chou, M. Y. X-ray studies of phonon softening in $\mathrm{TiSe}_{2}$. Phys. Rev. Lett. 86, 3799 (2001).

46. Chen, $\mathrm{P}$. et al. Hidden order and dimensional crossover of the charge density waves in $\mathrm{TiSe}_{2}$. Sci. Rep. 6, 37910 (2016).

47. Chen, P. Charge density wave transition in single-layer titanium diselenide. Nat Commun. 6, 8943 (2015).

48. Rossnagel, K. On the origin of charge-density waves in select layered transitionmetal dichalcogenides. J. Phys.: Condens. Matter 23, 213001 (2011).

49. Rossnagel, K. Suppression and emergence of charge-density waves at the surfaces of layered $1 \mathrm{~T}-\mathrm{TiSe}_{2}$ and $1 \mathrm{~T}-\mathrm{TaS}_{2}$ by in situ RB deposition. N. J. Phys. 12, 125018 (2010).

50. Rossnagel, K., Kipp, L. \& Skibowski, M. Charge-density-wave phase transition in $1 \mathrm{~T}-\mathrm{TiSe}_{2}$ : excitonic insulator versus band-type Jahn-Teller mechanism. Phys. Rev. $B$ 65, 235101 (2002).

51. Anderson, O., Manzke, R. \& Skibowski, M. Three-dimensional and relativistic effects in layered 1T-TiSe 2 . Phys. Rev. Lett. 55, 2188 (1985).

52. Traum, M. M., Margaritondo, G., Smith, N. V. \& Rowe, J. E. TiSe ${ }_{2}$ : semiconductor semimetal, or excitonic insulator. Phys. Rev. B 17, 1836 (1978).

53. Stoffel, N. G., Kevan, S. D. \& Smith, N. V. Experimental band structure of $1 \mathrm{~T}-\mathrm{TiSe}_{2}$ in the normal and charge-density-wave phases. Phys. Rev. B 31, 8049 (1985)

54. Pillo, T., Hayoz, J., Berger, H., Levy, F., Schlapbach, L. \& Aebi, P. Photoemission of bands above the Fermi level: the excitonic insulator phase transition in $1 \mathrm{~T}-\mathrm{TiSe}_{2}$ Phys. Rev. B 61, 16213 (2000).

55. Kidd, T. E., Miller, T. \& Chou, M. Y. Electron-hole coupling and the charge density wave transition in $\mathrm{TiSe}_{2}$. Phys. Rev. Lett. 88, 226402 (2002).

56. Rasch, J. C. E., Stemmler, T., Muller, B., Dudy, L. \& Manzke, R. 1T-TiSe 2 : semimetal or semiconductor? Phys. Rev. Lett. 101, 237602 (2008).

57. Li, G. Semimetal-to-semimetal charge density wave transition in $1 \mathrm{~T}-\mathrm{TiSe}_{2}$. Phys. Rev. Lett. 99, 027404 (2007)

58. Bianco, R., Calandra, M. \& Mauri, F. Electronic and vibrational properties of $\mathrm{TiSe}_{2}$ in the charge-density-wave phase from first principles. Phys. Rev. B 92, 094107 (2015).

59. Cazzaniga, M. Ab initio many-body effects in $\mathrm{TiSe}_{2}$ : a possible excitonic insulator scenario from GW band-shape renormalization. Phys. Rev. B 85, 195111 (2012).

60. van Schilfgaarde, M., Kotani, T. \& Faleev, S. V. Adequacy of approximations in GW theory. Phys. Rev. B 74, 245125 (2006).

61. Holm, B. Fully self-consistent GW self-energy of the electron gas. Phys. Rev. B $\mathbf{5 7}$ 2108 (1998).

62. Shirley, E. L. Self-consistent gw and higher-order calculations of electron states in metals. Phys. Rev. B 54, 7758 (1996).

63. Belashchenko, K. D., Antropov, V. P. \& Zein, N. E. Self-consistent local GW method application to $3 d$ and $4 d$ metals. Phys. Rev. B 73, 073105 (2006).

64. Tamme, D., Schepe, R. \& Henneberger, K. Comment on self-consistent calculations of quasiparticle states in metals and semiconductors. Phys. Rev. Lett. 83, 241 (1999).

65. Caruso, F., Dauth, M., van Setten, M. J. \& Rinke, P. Benchmark of GW approaches for the GW100 test set. J. Chem. Theory Comput. 12, 5076-5087 (2016).

66. Zhang, Z. et al. Direct photoluminescence probing of ferromagnetism in monolayer two-dimensional $\mathrm{CrBr}_{3}$. Nano Lett. 19, 3138-3142 (2019).

67. Molina-Sánchez, A., Catarina, G., Sangalli, D. \& Fernández-Rossier, J. Magnetooptical response of chromium trihalide monolayers: chemical trends. J. Mater Chem. C 8, 8856-8863 (2020).

68. Albrecht, S., Reining, L., Del Sole, R. \& Onida, G. Ab initio calculation of excitonic effects in the optical spectra of semiconductors. Phys. Rev. Lett. 80, 4510-4513 (1998).

69. Rohlfing, M. \& Louie, S. G. Electron-hole excitations and optical spectra from first principles. Phys. Rev. B 62, 4927-4944 (2000).

70. Deguchi, D., Sato, K., Kino, H. \& Kotani, T. Accurate energy bands calculated by the hybrid quasiparticle self-consistent GW method implemented in the ecalj package. Jpn. J. Appl. Phys. 55, 051201 (2016). 
71. Chantis, A. N., van Schilfgaarde, M. \& Kotani, T. Ab-initio prediction of conduction band spin splitting in zincblende semiconductors. Phys. Rev. Lett. 96, 086405 (2006).

72. Bhandari, C., van Schilfgaarde, M., Kotani, T. \& Lambrecht, W. R. L. All-electron quasiparticle self-consistent $\mathrm{GW}$ band structures for $\mathrm{SrTiO}_{3}$ including lattice polarization corrections in different phases. Phys. Rev. Mater. 2, 013807 (2018).

73. Cunningham, B., Grüning, M., Azarhoosh, P., Pashov, D. \& van Schilfgaarde, M. Effect of ladder diagrams on optical absorption spectra in a quasiparticle selfconsistent GW framework. Phys. Rev. Mater. 2, 034603 (2018).

74. Kutepov, A. L. Electronic structure of $\mathrm{Na}, \mathrm{K}, \mathrm{Si}$, and LiF from self-consistent solution of Hedin's equations including vertex corrections. Phys. Rev. B 94, 155101 (2016).

75. Kotani, T. Optimized effective potential method for solids with exact exchange and exact RPA correlation. J. Phys.: Condens. Matter 10, 9241 (1998).

76. Grüning, M., Marini, A. \& Rubio, A. Density functionals from many-body perturbation theory: the band gap for semiconductors and insulators. J. Chem. Phys. 124, 154108 (2006)

77. Olsen, T. \& Thygesen, K. S. Extending the random-phase approximation for electronic correlation energies: the renormalized adiabatic local density approximation. Phys. Rev. B 86, 081103 (2012).

78. Andersen, O. K. Linear methods in band theory. Phys. Rev. B 12, 3060-3083 (1975).

79. Faleev, S. V., van Schilfgaarde, M. \& Kotani, T. All-electron self-consistent GW approximation: application to Si, MnO, and NiO. Phys. Rev. Lett. 93, 126406 (2004).

80. Hirata, S. \& Head-Gordon, M. Time-dependent density functional theory within the tamm-dancoff approximation. Chem. Phys. Lett. 314, 291-299 (1999).

81. Myrta, G., Marini, A. \& Gonze, X. Exciton-plasmon states in nanoscale materials breakdown of the tamm- dancoff approximation. Nano Lett. 9, 2820-2824 (2009).

\section{ACKNOWLEDGEMENTS}

M.I.K., A.N.R., and S.A. are supported by the ERC Synergy Grant, project 854843 FASTCORR (Ultrafast dynamics of correlated electrons in solids). M.v.S. and D.P. were supported in the late stages of this work by the U.S. Department of Energy, Office of Science, Basic Energy Sciences under award FWP ERW7906. We acknowledge PRACE for awarding us access to Irene-Rome hosted by TGCC, France and Juwels Booster and Cluster, Germany. This work was also partly carried out on the Dutch national e-infrastructure with the support of SURF Cooperative.

\section{AUTHOR CONTRIBUTIONS}

M.I.K. and M.v.S. conceived the main theme of the work. S.A., D.P., and M.v.S. have carried out the calculations. All authors have contributed to the writing of the paper and the analysis of the data.

\section{COMPETING INTERESTS}

The authors declare no competing interests.

\section{ADDITIONAL INFORMATION}

Correspondence and requests for materials should be addressed to Swagata Acharya.

Reprints and permission information is available at http://www.nature.com/ reprints

Publisher's note Springer Nature remains neutral with regard to jurisdictional claims in published maps and institutional affiliations.

Open Access This article is licensed under a Creative Commons cc) Attribution 4.0 International License, which permits use, sharing, adaptation, distribution and reproduction in any medium or format, as long as you give appropriate credit to the original author(s) and the source, provide a link to the Creative Commons license, and indicate if changes were made. The images or other third party material in this article are included in the article's Creative Commons license, unless indicated otherwise in a credit line to the material. If material is not included in the article's Creative Commons license and your intended use is not permitted by statutory regulation or exceeds the permitted use, you will need to obtain permission directly from the copyright holder. To view a copy of this license, visit http://creativecommons. org/licenses/by/4.0/.

(c) The Author(s) 2021 